\title{
Epidemiology of Febrile Neutropenia Episodes with Gram-Negative Bacteria Infection in Patients Who Have Undergone Chemotherapy for Hematologic Malignancies: A Retrospective Study of 10 Years' Data from a Single Center
}

This article was published in the following Dove Press journal: Infection and Drug Resistance

\author{
Yunxiang Zhang (D) ${ }^{1,2, *}$ \\ Yu Zheng ${ }^{l, 2, *}$ \\ Fangyi Dong ${ }^{1,2, *}$ \\ Handong $\mathrm{Ma}^{3}$ \\ Liping Zhu' \\ Dake Shi iD 4 \\ Xiaoyang $\mathrm{Li}^{1,2}$ \\ Junmin $\mathrm{Li}^{1,2}$ \\ Jiong $\mathrm{Hu}^{\mathrm{I}, 2}$
}

'Department of Hematology, Ruijin Hospital, Shanghai Jiao Tong University School of Medicine, Shanghai, People's Republic of China; ${ }^{2}$ State Key Laboratory of Medical Genomics, Shanghai Institute of Hematology, Ruijin Hospital, Shanghai Jiao Tong University School of Medicine, Shanghai, People's Republic of China; ${ }^{3} J o h n$ Hopcroft Center for Computer Science, Department of Computer Science and Engineering, Shanghai Jiao Tong University, Shanghai, People's Republic of China; ${ }^{4}$ Department of Clinical Microbiology, Ruijin Hospital, Shanghai Jiao Tong University School of Medicine, Shanghai, People's Republic of China

*These authors contributed equally to this work

Correspondence: Junmin Li; Jiong $\mathrm{Hu}$ Tel +862I 64370045665258

Fax +86-2I-64374756

Email drlijunmin@I26.com;

hj10709@rjh.com.cn
Background: The epidemiology of Gram-negative bacteria in patients with febrile neutropenia (FN) and their susceptibility to initial empirical antibiotic therapy is key to successful treatment during the treatment of hematologic malignancies.

Methods: A retrospective study was conducted. Patients with FN and confirmed laboratory results of Gram-negative bacteria infections were included. If no direct sensitivity of the identified pathogen to the initially prescribed antibiotic regimen was confirmed, it was defined as inappropriate initial antibiotic treatment (IIAT).

Results: A total of 247 patients with FN were proven to be infected with Gram-negative bacteria, and 200 were diagnosed with acute leukemia. The most commonly detected bacteria were Escherichia coli (40\%), Klebsiella pneumoniae (20\%), and Pseudomonas aeruginosa $(11 \%)$. In sum, 176 patients were classified as IIAT. The mortality rate in the IIAT group was significantly higher $(37.7 \%$ vs $23.9 \%, P=0.038)$. With monotherapy as empirical treatment, high possibility of IIAT with fluoroquinolones (52\%) and cephalosporins (35\%) was detected, while more sensitivity to carbapenems (16\%) and glycopeptides antibiotics (19\%) was noticed. With combined treatment, cephalosporins/carbapenems had with the lowest percentage of IIAT (18\%).

Conclusion: In conclusion, inappropriate initial empirical antibiotic treatments were associated with higher mortality in patients with hematologic malignancies. The current empirical antibiotic regimen needs to be further optimized

Keywords: febrile neutropenia, Gram-negative bacteria, hematologic malignancy, empirical antibiotic treatment, inappropriate initial antibiotic treatment

\section{Introduction}

Intensive chemotherapy/radiotherapy in cancer, especially with hematologic malignancies, causes cellular injury and suppression of inflammatory responses, which increase risks of neutropenia and febrile episodes. ${ }^{1}$ The definition of febrile neutropenia (FN) may vary in different institutes and countries. Absolute neutrophil count (ANC) $<10^{9} / \mathrm{L}$ is considered neutropenia, with $\mathrm{ANC}<0.5 \times 10^{9} / \mathrm{L}$ or $<10^{9} / \mathrm{L}$ that is expected to decrease to $<0.5 \times 10^{9} / \mathrm{L}$ in the next 48 hours considered severe neutropenia, while $\mathrm{ANC}<0.1 \times 10^{9}$ is referred as profound neutropenia. Febrile episodes are 
usually defined as oral temperature $>38.3^{\circ} \mathrm{C}$ or two consecutive readings $>38.0^{\circ} \mathrm{C}$ lasting more than 1 hour. $\mathrm{FN}$ most often occurs during the initial diagnosis and treatment of malignancies. Although there is the possibility of noninfection-caused FN, most episodes are caused by infections. $\mathrm{FN}$ is a clinical emergency that requires prompt management. Despite advances in therapy in recent years, FN remains a common complication in chemotherapy causing serious clinical results, including death.

Administration of empirical antibacterial therapy has been successful in the management of FN since launching 50 years ago. Strategies for evaluation and treatment for FN in cancer patients have been well established and addressed in several international clinical guidelines. ${ }^{2-4}$ Wide application of broad-spectrum antibiotics has effectively decreased the mortality of FN patients. It was reported that the mortality rate of hospitalized FN patients in the US was only $9.5 \%$ in $2000 .{ }^{5}$ However, the general death rate of patients with FN can still reach $20 \%-$ $30 \%{ }^{4}$ A recent study showed that uncommon Gramnegative bacteria occur in increasing numbers in many infections, such as urinary tract infections. ${ }^{6}$ Patients with malignancy and other immunocompromising conditions are more likely to be infected with opportunistic Gramnegative bacteria. ${ }^{7}$ In addition, the usage of broadspectrum antibiotics has increased antibacterial resistance in bacteria. Lately, multidrug resistant (MDR) Gramnegative bacteria have been more often identified as causative agents of FN-related infections, which makes the choice of antibiotic-treatment regimen more complicated. Also, years of empirical antibiotic treatment have caused shift in infection pathogens from mainly Gram-negative to more Gram-positive bacteria. Currently, Gram-positive bacteria is a little more common in FN-related infection; however, Gram-negative bacteria still account for $40 \%-$ $50 \%$ of pathogens identified in FN patients. Meanwhile, therapy for Gram-positive bacteria is easier, since more drugs are available and novel antibiotics are emerging. ${ }^{8}$ Furthermore, patients with Gram-negative bacteria infection have higher risk of sepsis and death. It has been proven that patients with Gram-negative bacteria infection have worse prognosis, with mortality of $18 \%$ in Gramnegative and 5\% in Gram-positive bacteremia. ${ }^{9}$ Due to the high prevalence of antibiotic resistance and mortality in Gram-negative bacteria infection, the corresponding treatment regimen is still of great clinical importance.

The change in the bacterial spectrum has raised the question of modifying treatment regimens for appropriate treatment of patients. There have been a number of related studies $^{10-12}$ addressing empirical antibiotic treatment in FN; however, it has been suggested that epidemiology and resistance patterns in local hospitals should be considered when choosing empirical antibiotic treatments, as the optimal treatment strategy might vary in different local settings. In the current study, we aimed to study the epidemiology of Gram-negative bacteria in patients with FN during chemotherapy for hematologic malignancies and their susceptibility to initial empirical antibiotic treatments.

\section{Methods}

\section{Study Design and Patient Cohort}

This retrospective-cohort study was performed by screening a database of patients who underwent initial chemotherapy due to hematologic malignancies from January 2007 to December 2017 in the Department of Hematology, Shanghai Jiao Tong University School of Medicine. The study was approved by the Ruijin Hospital Ethics Committee (Shanghai Jiao Tong University School of Medicine), with ethics-approval reference number 2014 65. The review board exempted the need for informed consent, because this was retrospective study and did not affect patients.

Patients with records of FN and confirmed laboratory tests of Gram-negative bacteria infections were included. The empirical antibiotic-treatment regimens applied were compared to the results of antibioticsusceptibility/resistance tests to identify the occurrence of inappropriate initial antibiotic treatment (IIAT). Correlations between IIAT,with in-hospital death and empirical treatment regimen with a chance of IIAT were statistically analyzed. The database contained all records of patients, including clinical data, treatment records, laboratory test results of pathogens and antibiotic susceptibility/resistance.

Screening of study subjects was performed. Inclusion criteria were: initially diagnosed with hematologic malignancies, including acute lymphoblastic leukemia, acute myeloid leukemia, multiple myeloma, myelodysplastic syndromes, Hodgkin's lymphoma, and non-Hodgkin's lymphoma; and report of FN, defined as total neutrophil (granulocyte) count $<1,000 / \mu \mathrm{L}$. Exclusion criteria were missing data on infected strains/antibiotic treatment, pathogens other than Gram-negative bacteria, Initial empirical therapy focusing on antiviral/fungal treatment, and being accompanied by other immunological diseases. 


\section{Antimicrobial-Susceptibility Testing}

Clinical samples - blood, sputum, throat swab, midstream urine, hydrothorax, and ascites - were collected by conventional methods recommended in the clinic. Samples were processed according to the standard operating procedure of our institution. All isolates were identified by the VITEK 2 compact system and VITEK 2 Gram-negative identification card (BioMérieux, Marcy-l'Étoile, France). The microbiology laboratory performed antimicrobialsusceptibility testing of the Gram-negative bacterial isolates based on minimum inhibitory concentration. More detailed methods have been described in our previous studies. ${ }^{13,14}$

\section{Definition of IIAT and Data Collection}

Empirical antibiotic treatment was classified as being appropriate if direct sensitivity of the identified pathogen to the initially prescribed antibiotic regimen was confirmed based on in vitro susceptibility testing, while confirmed antibiotic resistance and no direct testing of prescribed antibiotics were considered IIAT. Baseline patient characteristics and clinical data collected included age, sex, comorbidities, such as diabetes and manifestations of neutropenia and/or fever, and complications (including liver damage, respiratory failure, renal insufficiency, bleeding, heart failure, and requirement of ventilator support).

\section{Statistical Analysis}

Patients were allocated into IIAT and non-IIAT groups according to appropriateness of empirical antibiotic regimens. Baseline characteristics and clinical data were compared between the two groups. Descriptive statistics were used to analyze the chances of IIAT in cases with different bacterial infections and various initial empirical antibiotic-treatment choices. Continuous variables are reported as means $\pm \mathrm{SD}$. Student's $t$-test was used to compare normally distributed data between the two groups, and the Mann-Whitney $U$ test was used to analyze abnormally distributed data. Categorical data are expressed as frequency and percentage, and $\chi^{2}$ was used to determine differences between groups. All tests were two-tailed, and $P-<0.05$ was considered statistically significant.

\section{Results}

\section{Patient Characteristics and Clinical Manifestations}

A total of 276 episodes of FN and complete microbiologytest results were included, 29 of which were different specimens from the same patient. As such, 247 patients were included in the study: 71 patients were identified as treated with an appropriate initial antibiotic regimen (nonIIAT) and 176 classified as IIAT. Baseline characteristics and clinical data were extracted and compared between IIAT and non-IIAT patients. As shown in Table 1, the IIAT group contained a higher percentage of female patients. No other characteristics or clinical manifestations, including underlying diseases and treatment type,o showed a significant difference between the two groups. We also collected some usual complications of Gram-negative bacteria infection. The severity of complications was documented according to Common Terminology Criteria for Adverse Events 4.0, and complications >grade 2 were listed. All deaths caused by infection and related adverse events were collected. There were 83 hospital deaths reported, with a significantly $(P=0.038)$ higher death rate (66 of 176, 37.7\%) in IIAT patients than non-IIAT patients (17 of $71,23.9 \%)$.

\section{Gram-Negative Bacteria Species}

The laboratory microbiology-test results identified $>30$ species of Gram-negative bacteria as causative agents of infection. Pathogens identified in multiple patients are summarized in Table 2. The most commonly detected species were Escherichia coli (40\%), Klebsiella pneumoniae (20\%), Pseudomonas aeruginosa (11\%), and Stenotrophomonas maltophilia (9\%). In addition, $>20$ bacterial species were detected in individual cases. A few cases were identified with infection by multiple bacteria, including E. coli-nonVibrio cholerae O1, E. coli-Bacteroides fragilis, $K$. pneumoniae-P. aeruginosa, $K$. pneumoniaeStaphyphila maltophilia, Acinetobacter lwoffii-E. coli, and $P$. aeruginosa-K. pneumoniae.

Antibiotic susceptibility/resistance of infected bacteria was tested (Tables 2 and 3). For the most common pathogen strains - E. coli, $K$. pneumoniae, and $P$. aeruginosa - susceptibility to initial empirical treatment was $46.2 \%, 38.8 \%$, and $55.6 \%$, respectively. Confirmed resistance to initial empirical treatment was $31.2 \%, 22.4 \%$, and $7.4 \%$, respectively, for these species. In the bacteria detected, Sphingomonas paucimobilisand $P$. aeruginosa tended to be sensitive to the initial treatment, while resistance was mostly identified for Enterobacter cloacae, E. coli, and K. pneumoniae. 
Table I Comparison of baseline characteristics and clinical data between patients with different initial empirical antibiotic treatments

\begin{tabular}{|c|c|c|c|}
\hline & Non-IIAT $(n=7 I)$ & IIAT $(n=\mid 76)$ & $P$-value \\
\hline Age, years (mean \pm SD) & $38.6 \pm 14.7$ & $38.8 \pm 15.8$ & 0.963 \\
\hline $\operatorname{Sex}(M / F)$ & $51 / 20$ & $99 / 77$ & 0.023 \\
\hline Duration of fever (days), median (range) & $3(0-35)$ & $4(0-54)$ & 0.179 \\
\hline Underlying disease & & & 0.831 \\
\hline Acute lymphoblastic leukemia, $n$ (\%) & 25 (35.2\%) & $6 \mathrm{I}(34.7 \%)$ & \\
\hline Acute myeloid leukemia, n (\%) & $31(43.7 \%)$ & $83(47.1 \%)$ & \\
\hline Other hematologic malignancy ${ }^{\mathrm{a}}, \mathrm{n}(\%)$ & $15(21.1 \%)$ & $32(18.2 \%)$ & \\
\hline Treatment type $^{b}$ & & & 0.592 \\
\hline Hematopoietic stem-cell transplantation, n (\%) & $27(38.0 \%)$ & $71(40.3 \%)$ & \\
\hline High-dose chemotherapy, n (\%) & $33(46.5 \%)$ & $86(48.9 \%)$ & \\
\hline Other chemotherapy, n (\%) & II (I5.5\%) & $19(10.8 \%)$ & \\
\hline \multicolumn{4}{|l|}{ Complications (grade 2-4) } \\
\hline Ventilator support, n (\%) & $6(8.5 \%)$ & $13(7.4 \%)$ & 0.795 \\
\hline Liver damage, $\mathrm{n}(\%)$ & $23(32.4 \%)$ & 78 (44.6\%) & 0.079 \\
\hline Respiratory failure, n (\%) & 8 (II.3\%) & $33(18.9 \%)$ & 0.148 \\
\hline Renal insufficiency, n (\%) & $47(66.2 \%)$ & $105(60 \%)$ & 0.365 \\
\hline Bleeding, n (\%) & $26(36.6 \%)$ & $67(38.3 \%)$ & 0.807 \\
\hline Diabetes, n (\%) & $3(4.2 \%)$ & $5(2.9 \%)$ & 0.693 \\
\hline Heart failure, n (\%) & $4(5.6 \%)$ & $18(10.3 \%)$ & 0.247 \\
\hline Death, n (\%) & 17 (23.9\%) & 66 (37.7\%) & 0.038 \\
\hline
\end{tabular}

Notes: ${ }^{a}$ Other hematologic diseases included multiple myeloma, myelodysplastic syndromes, non-Hodgkin's lymphoma, and Hodgkin's lymphoma; ${ }^{b}$ treatment regimens and classification summarized in Supplementary Table I.

Table 2 Gram-negative bacteria pathogens and susceptibility to initial empirical treatment identified in febrile neutropenia patients

\begin{tabular}{|c|c|c|c|c|}
\hline & Patients, n (\%) & Death, n (\%) & $\begin{array}{l}\text { Sensitive to initial } \\
\text { treatment, n (\%) }\end{array}$ & $\begin{array}{l}\text { Confirmed resistance to } \\
\text { initial treatment (\%) }\end{array}$ \\
\hline E. coli & $93(40.0)$ & $22(43.0)$ & $43(46.2)$ & $29(31.2)$ \\
\hline K. pneumoniae & $49(20.0)$ & $21(42.9)$ & $19(38.8)$ & II (22.4) \\
\hline$P$. aeruginosa & $27(11.0)$ & 7 (25.9) & I5 (55.6) & $2(7.4)$ \\
\hline S. maltophilia & $22(9.0)$ & II (50) & $4(18.2)$ & $4(18.2)$ \\
\hline E. cloacae & $8(3.3)$ & $2(25)$ & $I(12.5)$ & $4(50)$ \\
\hline S. paucimobilis & $7(2.9)$ & 0 & $5(71.4)$ & I (I4.3) \\
\hline A. baumannii & $5(2.0)$ & $5(100)$ & I (20) & NA \\
\hline Pantoea & $3(1.2)$ & 0 & I (33.3) & NA \\
\hline Clostridium falciparum & $2(0.8)$ & I (50) & 0 & I (50) \\
\hline Bacillus cereus & $2(0.8)$ & I (50) & 0 & NA \\
\hline Burkholderia cepacia & $2(0.8)$ & $2(100)$ & 0 & NA \\
\hline
\end{tabular}

\section{Empirical Antibiotic-Treatment Regimen and Probability of IIAT}

In the study center, the empirical antibiotic regimens were chosen according to European Society of Medical Oncology guidelines for management of $\mathrm{FN}^{4}$ The most commonly applied monotherapy regimens were carbapenems, cephalosporin, glycopeptide antibiotics, and fluoroquinolones. As shown in Table 4, there was a high possibility $(52 \%$ and $35 \%)$ of IIAT when treating with fluoroquinolones and cephalosporin, respectively, while more sensitivity to carbapenems (IIAT 16\%) and glycopeptide antibiotics (IIAT 19\%) was noticed. In combinedtreatment regimens, cephalosporins-carbapenems, fluoroquinolones-metronidazole, and fluoroquinolones-cephalosporins were the most commonly used. Among those, cephalosporins-carbapenems showed the lowest rate of IIAT (18\%), while the other two had relatively high rates of IIAT ( $50 \%$ and $33 \%$, respectively). The most 
Table 3 Bacteria prone to sensitivity/resistance to initial empirical antibiotic treatment

\begin{tabular}{|l|l|l|l|l|l|}
\hline & Patients, $\mathbf{n}$ & $\begin{array}{l}\text { Sensitive to initial } \\
\text { treatment, n (\%) }\end{array}$ & & Patients, n & $\begin{array}{l}\text { Confirmed resistance to } \\
\text { initial treatment, n (\%) }\end{array}$ \\
\hline $\begin{array}{l}\text { S. paucimobilis } \\
\text { P. aeruginosa }\end{array}$ & 7 & $5(71.4)$ & E. cloacae & 7 & $4(57.1)$ \\
Pantoea & 27 & $15(55.6)$ & E. coli & 93 & $29(31.2)$ \\
E. coli & 93 & $I(50)$ & K. pneumoniae & 49 & $11(22.4)$ \\
K. pneumoniae & 49 & $43(46.2)$ & S. maltophilia & 22 & $4(18.2)$ \\
A. baumannii & 5 & $19(38.8)$ & S. paucimobilis & 7 & I (14.3) \\
S. maltophilia & 22 & $1(20)$ & P. aeruginosa & 27 & $2(7.4)$ \\
\hline
\end{tabular}

Table 4 Empirical treatment with monotherapy/combined antibiotics and probability of IIAT

\begin{tabular}{|l|l|l|l|}
\hline & Patients (n) & IIAT (n) & IIAT \\
\hline Monotherapy & & & \\
B-lactamase inhibitors & 1 & 1 & $100 \%$ \\
Fluoroquinolones & 33 & 17 & $52 \%$ \\
Metronidazole & 14 & 7 & $50 \%$ \\
Oxazolidinones & 2 & 1 & $50 \%$ \\
Cephalosporin & 63 & 22 & $35 \%$ \\
Anti-Pseudomonas penicillin & 17 & 6 & $35 \%$ \\
Glycopeptide antibiotics & 42 & 8 & $19 \%$ \\
Carbapenems & 63 & 10 & $16 \%$ \\
Aminoglycosides & 12 & 1 & $8 \%$ \\
Tetracyclines & 3 & 0 & 0 \\
Macrolides & 1 & 0 & 0 \\
\hline Combined antibiotics & & & \\
Fluoroquinolones-metronidazole & 10 & 5 & $50 \%$ \\
Fluoroquinolones-cephalosporins & 9 & 3 & $33 \%$ \\
Anti-Pseudomonas penicillin- & 6 & 2 & $33 \%$ \\
glycopeptides & & & \\
Cephalosporins-carbapenems & 11 & 2 & $18 \%$ \\
Aminoglycosides-cephalosporins & 6 & 1 & $17 \%$ \\
Fluoroquinolones-glycopeptides & 6 & 1 & $17 \%$ \\
Carbapenems-metronidazole & 6 & 1 & $17 \%$ \\
Carbapenems-glycopeptides & 21 & 3 & $14 \%$ \\
Cephalosporins-glycopeptides & 15 & 2 & $13 \%$ \\
Fluoroquinolones-carbapenems & 10 & 1 & $10 \%$ \\
Aminoglycosides-glycopeptides & 5 & 0 & 0 \\
Aminoglycosides-carbapenems & 4 & 0 \\
Others & 18 & $28 \%$ \\
\hline
\end{tabular}

commonly used antibiotics (including monotherapies and combined regimens) are shown in Table 5. Empirical treatment with meropenem and combined therapy with imipenem-cilastatin-vancomycin were all appropriate. All other antibioticsshowed resistance of $33 \%-80 \%$.

\section{Discussion}

From January 2007 to December 2017 in the Department of Hematology, patients who underwent chemotherapy and hematopoietic stem-cell transplantation for hematologic malignancies experienced FN episodes, of whom 247 were proven to be infected by Gram-negative bacteria. Gram-negative bacteria accounted for $46 \%$ of FN pathogens detected (data not shown). A previous study in pediatric patients with hematologic malignancies showed that prolonged neutropenia and duration of hospitalization were associated with Gram-negative infection, especially carbapenem-resistant bacteremia. ${ }^{15}$ In our study, $81 \%$ of FN patients were diagnosed with acute leukemia and 98 (39.7\%) underwent stem-cell transplantation. The reason for these data may be that patients who underwent stemcell transplantation or induction and consolidation for acute leukemia were prescribed higher-dose chemotherapy, which may cause longer neutropenia.

Since FN is considered a clinical emergency that needs timely management, empirical antibacterial treatment is administered before assessment of infection pathogens. An inappropriate choice of treatment regimen would reduce response rate and result in higher mortality. In this study, initial empirical antibiotic treatments were detected to be appropriate for 71 patients, which means only about $30 \%$ of isolated Gram-negative bacteria were sensitive to the initial empirical treatment. Due to the higher load of MDR bacteria, FN patients with Gram-negative organism infection usually present with quick disease onset and multiple complications. In our study, more than half the patients progressed to renal insufficiency and a third suffered liver damage and bleeding. The mortality of the whole cohort was $33.6 \%$, which is higher than previously reported in cancer patients. ${ }^{9}$ A possible reason is that the subjects in this study were under treatment for hematologic malignancies, generally considered to be higher-risk. Also, IIAT patients had higher mortality (37.7\%) than non-IIAT patients $(23.9 \%)$, indicating a correlation between appropriate initial empirical treatment with poor prognosis and 
Table 5 Most commonly used antibiotics and confirmed resistance rate

\begin{tabular}{|l|l|l|l|l|}
\hline & Patients (n) & $\begin{array}{l}\text { Confirmed } \\
\text { sensitivity (n) }\end{array}$ & $\begin{array}{l}\text { Confirmed } \\
\text { resistance (n) }\end{array}$ & Resistance rate \\
\hline $\begin{array}{l}\text { Monotherapy } \\
\text { Meropenem }\end{array}$ & 12 & 12 & & \\
Cefepime & 8 & 4 & 4 & 0 \\
Moxifloxacin & 6 & 1 & 5 & $50 \%$ \\
Ceftazidime & 5 & 3 & 2 & $83 \%$ \\
Cefotaxime & 4 & 1 & 3 & $40 \%$ \\
\hline Combined antibotics & & & & $75 \%$ \\
Imipenem-cilastin & 10 & 6 & 4 & \\
Piperacillin-tazobactam & 8 & 5 & 3 & $30 \%$ \\
Moxifloxacin-metronidazole & 5 & 1 & 4 & $80 \%$ \\
Meropenem-tecolanin & 4 & 3 & 1 & $25 \%$ \\
Meropenem-vancomycin & 4 & 2 & 2 & $50 \%$ \\
Cefoperazone-sulbactam & 4 & 2 & 2 & $50 \%$ \\
Ceftazidime-vancomycin & 4 & 2 & 0 & $50 \%$ \\
Imipenem-cilastatin-vancomycin & 4 & 4 & 1 & 0 \\
Piperacillin-tazobactam-vancomycin & 3 & 1 & & $33 \%$ \\
\hline
\end{tabular}

higher mortality. These results suggest that current empirical antibiotic regimens need to be further optimized.

Gram-negative bacteria accounted for the major pathogens of $\mathrm{FN}$ in cancer patients in the early stage. Although Gram-positive organisms have been identified as more infectious pathogens in recent years, due to their more common drug resistance, Gram-negative pathogen are still an important concern in FN management. In our data, the most commonly detected species were E. coli, K. pneumoniae, $P$. aeruginosa, and $S$. maltophilia, with all strains identified having a certain degree of resistance to antibiotics. A previous single-center retrospective study showed that bloodstream infection was mostly caused by Gram-negative bacteria. ${ }^{16}$ A study in south India showed that Gram-negative bacilli accounted for $40 \%$ of $\mathrm{FN}$ pathogens, and E. coli, Acinetobacter baumannii, andK. pneumoniae were the most common species. ${ }^{17}$ A study in high-risk patients with hematologic malignancies showed that only $29.6 \%$ had positive microbiology cultures and Gram-negative bacilli was the most common (63.6\%) pathogen, with E. coli being the most frequent. ${ }^{18}$ These results are consistent with our identified Gram-negative bacteria species.

Carbapenem-resistant $K$. pneumoniae (CRKP) appears to be a serious problem in patients with hematologic malignancies, with high mortality. A study in high-risk hematologic patients showed that CRKP bacteremia developed mainly during neutropenia and caused high mortality (71\%) in acute myeloid leukemia patients. ${ }^{19}$ A retrospective analysis from a single center showed that Gram-negative bacteria were the predominant $(92 \%)$ pathogen and Klebsiella the most common species. MDR was common in these isolates, causing $15 \%$ mortality. Colistin was suggested as the salvage-therapy option. ${ }^{20}$ The resistance of K. pneumoniae to empirical treatment was also commonly detected in our study, indicating the importance of recognition of CRKP carriers in FN patients and timely empirical treatment with antibiotics active against CRKP.

There are two common strategies in empirical antibacterial treatment in $\mathrm{FN}^{21}$ An escalation begun with a more narrow-spectrum antibiotic choice that can be further broadened in cases of clinical deterioration or resistant pathogens has been identified. In contrast, de-escalation starts with an initial regimen covering drug-resistant pathogens, then de-escalating to specific antibiotic coverage after pathogen identification. ${ }^{1}$ Due to more common MDR in Gram-negative pathogens, the de-escalation strategy is recommended in cases of suspicion of Gram-negative bacteria infection. ${ }^{2}$ First-line empirical treatment depends on what is known about the patient. ${ }^{4}$ In FN episodes of hospitalized high-risk patients, it is usually recommended that an antipseudomonal $\beta$-lactam agent be used as initial empirical treatment. If risk of resistant-bacteria infection is suspected, addition of vancomycin, teicoplanin, linezolid, or daptomycin to the initial empirical therapy is considered. Also, colistin or tigecycline should be administered in cases of infections caused by KPC-producing Gram-negative bacteria. ${ }^{1,21}$ 
In a previous study, resistance to ciprofloxacin (5\%) appeared to be the lowest in this center. ${ }^{16}$ This result was different from our finding that resistance was mostly common to antibiotic categories of fluoroquinolones. Since the previous study was performed in a center where universal fluoroquinolone prophylaxis was not employed, these results may reflect the influence of regular fluoroquinolone application. As $\beta$ lactam antibiotics with a very broad spectrum, when combined with $\beta$-lactamase inhibitors, carbapenems show an even more extended spectrum of activity and seemto be an appropriate choice for treatment of MDR Gram-negative bacteria, which are a predominant source of drug resistance in $\mathrm{FN}^{22}$ Broadspectrum $\beta$-lactam has been suggested as initial empirical antibiotic treatment of FN in many countries, and a review of studies in Norway has proven its effectiveness. ${ }^{23} \beta$-lactam antibiotics are also first-choice agents in vulnerable patient populations, such as the elderly, children, and pregnant women. ${ }^{24} \mathrm{~A}$ meta-analysis of antipseudomonal $\beta$-lactam empirical monotherapy ${ }^{10}$ showed that imipenem-cilastatin gave the highest opportunity of successful treatment and lowest mortality. ${ }^{10}$ An imipenem and cefoperazone-sulbactam combination was shown to be efficient for most isolated Gram-negative bacteria and was suggested to be used as firstline empirical antibiotic therapy. ${ }^{18}$ These results seem to be consistent with our findings, indicating imipenem-cilastatin as an effective empirical treatment choice.

Most patients in the current study were treated with monotherapy. A systematic review of 68 studies of $\mathrm{FN}$ in pediatric patients who had undergone stem-cell transplantation ${ }^{25}$ showed that an aminoglycoside-containing combination therapy did not have a higher success rate or lower mortality compared with monotherapy, indicating a good choice of monotherapy may be sufficient in empirical antibiotic treatment of FN. An early study ${ }^{26}$ also showed that meropenem and piperacillin-tazobactam had comparable success rates, indicating the sufficiency of monotherapy. Therefore, a monotherapy seemed to be appropriate for initial empirical antibiotic treatment.

The current study has certain limitations. Due to its being retrospective, it may not have included significant missing data in the clinical records, which would cause bias in the results. Clinical characteristics, including risk stratification, considered an important risk factor in treatment success ${ }^{27}$ were not included in the analysis. We hope to address these questions in a more systematic manner in future studies. In conclusion, this retrospective review of 276 episodes of FN with confirmed Gram-negative bacteria infection showed that the most common pathogens were
E. coli, K. pneumoniae, P. aeruginosa, and S. maltophilia. Inappropriate initial empirical antibiotic treatments were associated with higher mortality.

\section{Ethical Approval}

All procedures involving human participants were in accordance with the ethical standards of the institutional and/or national research committee and with the 1964 Declaration of Helsinki and its later amendments or comparable ethical standards. This study was approved by the Ruijin Hospital Ethics Committee (Shanghai Jiao Tong University School of Medicine), and the ethicsapproval reference number is 2014 65. For this type of study, formal consent is not required. All authors confirmthat the data were anonymized and maintained with confidentiality. This article does not contain any studies with animals performed by any of the authors.

\section{Acknowledgments}

This work was supported by grants from the Shanghai ThreeYear Plan of the Key Subjects Construction (15GWZK0102, 16CR1034B), the National Key Research and Development Plan (2016YFC0902800), and the Shanghai Jiao Tong University School of Medicine Multi-Center Clinical Research Project (DLY201513). We would like to express our appreciation to the research assistants for their diligence and attentiveness to detail and the outstanding clinical care delivered by all staff. We would also like to show our gratitude to Shanghai Synyi Medical Technology Co Ltd for its assistance with data collection, cleanup, and analysis processes. Co-first authors: Yunxiang Zhang, Yu Zheng, Fangyi Dong.

\section{Disclosure}

The authors declare that they have no conflicts of interest in this work.

\section{References}

1. Alp S, Akova M. Management of febrile neutropenia in the era of bacterial resistance. Ther Adv Infect Dis. 2013;1(1):37-43. doi:10.1177/2049936113475610

2. Taplitz RA, Kennedy EB, Bow EJ, et al. Outpatient management of fever and neutropenia in adults treated for malignancy: American Society of Clinical Oncology and Infectious Diseases Society of America Clinical Practice Guideline Update. J Clin Oncol. 2018;36 (14); Jco2017776211.

3. Tissot F, Agrawal S, Pagano L, et al. ECIL-6 guidelines for the treatment of invasive candidiasis, aspergillosis and mucormycosis in leukemia and hematopoietic stem cell transplant patients. Haematologica. 2017;102(3):433-444. doi:10.3324/haematol.2016. 152900 
4. Klastersky J, de Naurois J, Rolston K, et al. Management of febrile neutropaenia: ESMO Clinical Practice Guidelines. Ann Oncol. 2016;27(suppl 5):v111-v8. doi:10.1093/annonc/mdw325

5. Kuderer NM, Dale DC, Crawford J, Cosler LE, Lyman GH. Mortality, morbidity, and cost associated with febrile neutropenia in adult cancer patients. Cancer. 2006;106(10):2258-2266. doi:10.1002/ (ISSN)1097-0142

6. Gajdacs M, Burian K, Terhes G. Resistance levels and epidemiology of non-fermenting gram-negative bacteria in urinary tract infections of inpatients and outpatients (RENFUTI): a 10-year epidemiological snapshot. Antibiotics (Basel). 2019;8:3.

7. Gajdacs M, Urban E. Epidemiological trends and resistance associated with stenotrophomonas maltophilia bacteremia: a 10-year retrospective cohort study in a tertiary-care hospital in hungary. Diseases. 2019;7(2):41. doi:10.3390/diseases7020041

8. Gajdacs M. The continuing threat of methicillin-resistant staphylococcus aureus. Antibiotics (Basel). 2019;8:2.

9. Klastersky J, Ameye L, Maertens J, et al. Bacteraemia in febrile neutropenic cancer patients. Int J Antimicrob Agents. 2007;30(Suppl 1):S51-S59. doi:10.1016/j.ijantimicag.2007.06.012

10. Horita N, Shibata Y, Watanabe H, Namkoong H, Kaneko T. Comparison of antipseudomonal beta-lactams for febrile neutropenia empiric therapy: systematic review and network meta-analysis. Clin Microbiol Infect. 2017;23(10):723-729. doi:10.1016/j.cmi.2017.03.024

11. Chen K, Wang Q, Pleasants RA, et al. Empiric treatment against invasive fungal diseases in febrile neutropenic patients: a systematic review and network meta-analysis. BMC Infect Dis. 2017;17(1):159. doi:10.1186/s12879-017-2263-6

12. Manji A, Lehrnbecher T, Dupuis LL, Beyene J, Sung L. A systematic review and meta-analysis of anti-pseudomonal penicillins and carbapenems in pediatric febrile neutropenia. Support Care Cancer. 2012;20(10):2295-2304. doi:10.1007/s00520-011-1333-3

13. Wang S, Zhao SY, Xiao SZ, et al. Antimicrobial resistance and molecular epidemiology of escherichia coli causing bloodstream infections in Three Hospitals in Shanghai, China. PLoS One. 2016;11(1):e0147740. doi:10.1371/journal.pone.0147740

14. Sun J, Xu Y, Yu Y, Ni Y. Accuracy of in vitro susceptibility tests for carbapenemase-producing Gram-negative bacteria. J Med Microbiol. 2015;64(6):620-622. doi:10.1099/jmm.0.000067

15. Vinker-Shuster M, Stepensky P, Temper V, Shayovitz V, Masarwa R, Averbuch D. Gram-negative bacteremia in children with hematologic malignancies and following hematopoietic stem cell transplantation: epidemiology, resistance, and outcome. J Pediatr Hematol Oncol. 2019;41(8):e493-e8. doi:10.1097/MPH.0000000000001556

16. Conn JR, Catchpoole EM, Runnegar N, Mapp SJ, Markey KA. Low rates of antibiotic resistance and infectious mortality in a cohort of high-risk hematology patients: a single center, retrospective analysis of blood stream infection. PLoS One. 2017;12(5):e0178059. doi:10.1371/journal.pone.0178059
17. Babu KG, Lokanatha D, Lakshmaiah KC, et al. Bloodstream infections in febrile neutropenic patients at a tertiary cancer institute in South India: a timeline of clinical and microbial trends through the years. Indian J Med Paediatr Oncol. 2016;37(3):174-182. doi:10. 4103/0971-5851.190352

18. Lakshmaiah KC, Malabagi AS, Govindbabu SR, Sinha M, Jayashree RS. Febrile neutropenia in hematological malignancies: clinical and microbiological profile and outcome in high risk patients. $J$ Lab Physicians. 2015;7(2):116-120. doi:10.4103/0974-2727. 163126

19. Micozzi A, Gentile G, Minotti C, et al. Carbapenem-resistant Klebsiella pneumoniae in high-risk haematological patients: factors favouring spread, risk factors and outcome of carbapenem-resistant Klebsiella pneumoniae bacteremias. BMC Infect Dis. 2017;17(1):203. doi:10.1186/s12879-017-2297-9

20. Reddy R, Pathania S, Kapil A, Bakhshi S. Review of spectrum and sensitivity of bacterial bloodstream isolates in children with malignancy: a retrospective analysis from a single center. Indian J Cancer. 2014;51(4):425-427. doi:10.4103/0019-509X.175363

21. Escrihuela-Vidal F, Laporte J, Albasanz-Puig A, Gudiol C. Update on the management of febrile neutropenia in hematologic patients. Rev Esp Quimioter. 2019;32(Suppl 2):55-58.

22. CADTH Rapid Response Reports. Carbapenems for Multi-Drug Resistant Infections: A Review of Guidelines. Ottawa, ON: Canadian Agency for Drugs and Technologies in Health Copyright (c) 2016 Canadian Agency for Drugs and Technologies in Health; 2016.

23. Torfoss D, Hoiby EA, Holte H, Kvaloy S. The Norwegian experience with penicillin $\mathrm{G}$ plus an aminoglycoside as initial empiric therapy in febrile neutropenia; a review. Acta Oncol. 2012;51(4):433-440. doi:10.3109/0284186X.2011.633931

24. Gajdacs M. The concept of an ideal antibiotic: implications for drug design. Molecules. 2019;24:5. doi:10.3390/molecules24050892

25. Robinson PD, Lehrnbecher T, Phillips R, Dupuis LL, Sung L. Strategies for empiric management of pediatric fever and neutropenia in patients with cancer and hematopoietic stem-cell transplantation recipients: a systematic review of randomized trials. $J$ Clin Oncol. 2016;34(17):2054-2060. doi:10.1200/JCO.2015. 65.8591

26. Sezgin G, Acipayam C, Ozkan A, Bayram I, Tanyeli A. Meropenem versus piperacillin-tazobactam as empiric therapy for febrile neutropenia in pediatric oncology patients. Asian Pac J Cancer Prev. 2014;15(11):4549-4553. doi:10.7314/APJCP.2014.15.11.4549

27. Haeusler GM, Sung L, Ammann RA, Phillips B. Management of fever and neutropenia in paediatric cancer patients: room for improvement? Curr Opin Infect Dis. 2015;28(6):532-538. doi:10.1097/QCO.0000000000000208
Infection and Drug Resistance

\section{Publish your work in this journal}

Infection and Drug Resistance is an international, peer-reviewed openaccess journal that focuses on the optimal treatment of infection (bacterial, fungal and viral) and the development and institution of preventive strategies to minimize the development and spread of resistance. The journal is specifically concerned with the epidemiology of antibiotic resistance and the mechanisms of resistance development and diffusion in both hospitals and the community. The manuscript management system is completely online and includes a very quick and fair peerreview system, which is all easy to use. Visit http://www.dovepress.com/ testimonials.php to read real quotes from published authors. 